\title{
Critical ruptures in a bundle of slowly relaxing fibers
}

\author{
K. Kovács, ${ }^{1}$ S. Nagy, ${ }^{1}$ R. C. Hidalgo, ${ }^{2}$ F. Kun, ${ }^{1, *}$ H. J. Herrmann, ${ }^{3}$ and I. Pagonabarraga ${ }^{4}$ \\ ${ }^{1}$ Department of Theoretical Physics, University of Debrecen, P. O. Box 5, H-4010 Debrecen, Hungary \\ ${ }^{2}$ AMADE, Departament de Física, Universitat de Girona, Avinguda Montilivi s/n, 17001-Girona, Spain \\ ${ }^{3}$ Computational Physics, IfB, HIF, E12, ETH, Hoenggerberg, 8093 Zürich, Switzerland \\ ${ }^{4}$ Departament de Física Fonamental, Universitat de Barcelona, Carrer Martí i Franqués 1, E-08028 Barcelona, Spain
}

(Received 7 September 2007; revised manuscript received 17 January 2008; published 3 March 2008)

We study the damage enhanced creep rupture of disordered materials by means of a fiber bundle model. Broken fibers undergo a slow stress relaxation modeled by a Maxwell element whose stress exponent $m$ can vary in a broad range. Under global load sharing we show that due to the strength disorder of fibers, the lifetime $t_{f}$ of the bundle has sample-to-sample fluctuations characterized by a log-normal distribution independent of the type of disorder. We determine the Monkman-Grant relation of the model and establish a relation between the rupture life $t_{f}$ and the characteristic time $t_{m}$ of the intermediate creep regime of the bundle where the minimum strain rate is reached, making possible reliable estimates of $t_{f}$ from short term measurements. Approaching macroscopic failure, the deformation rate has a finite time power law singularity whose exponent is a decreasing function of $m$. On the microlevel the distribution of waiting times is found to have a power law behavior with $m$-dependent exponents different below and above the critical load of the bundle. Approaching the critical load from above, the cutoff value of the distributions has a power law divergence whose exponent coincides with the stress exponent of Maxwell elements.

DOI: 10.1103/PhysRevE.77.036102

\section{INTRODUCTION}

Under high steady stresses, materials often show time dependent deformation and failure called creep rupture [1,2], which substantially limits their application in construction components [3]. In recent years the application of statistical physics in data evaluation of experiments and in theoretical approaches has provided insight into the time dependent rupture of disordered materials ranging from biological matter $[4,5]$ through construction materials $[6-8]$ to the behavior of fault zones responsible for the emergence of earthquakes $[1,2,9,10]$. It has been pointed out that in the case of high disorder the macroscopic failure event is preceded by an acceleration of microcracking resulting in a power law finite time singularity of several characteristic quantities of the fracturing system $[2,11-15]$. The acceleration process can be monitored experimentally by the acoustic emission technique $[14,16]$, which also addresses the possibility of forecasting the imminent failure $[8,13,17]$.

One of the most important theoretical approaches to the damage enhanced creep of materials with disordered microstructure is the fiber bundle model (FBM) with fibers having time dependent rheological behavior [18-25]. It has been shown in the framework of FBM that depending on the external load the creeping system may undergo a damage process and has an infinite lifetime, or breaks macroscopically in a finite time [18-22]. Between the two regimes a transition occurs at a critical load which is equal to the static tensile strength of the material. At macroscopic rupture $t_{f}$, the deformation rate $\dot{\varepsilon}$ was found to have a power law acceleration $\dot{\varepsilon} \sim\left(t_{f}-t\right)^{-\gamma}$ with an exponent $\gamma$ fluctuating about one $[8,18]$.

\footnotetext{
*feri@dtp.atomki.hu
}

PACS number(s): 46.50.+a, 62.20.M-, 46.35.+z

In the present paper we study the creep rupture process of a bundle of linearly elastic fibers where time dependence is introduced by the slow stress relaxation of broken fibers modeled by Maxwell elements. Our goal is to characterize how the system approaches macroscopic failure and derive relations of observables that can be used to forecast the imminent failure event. On the macrolevel we derive the Monkman-Grant relation of the model which expresses the lifetime $t_{f}$ as a function of the minimum strain rate $\dot{\varepsilon}$ of the creeping system. We characterize the sample-to-sample fluctuations of $t_{f}$ and demonstrate that for single samples $t_{f}$ has a universal relation with the characteristic time of the intermediate creep $t_{m}$. Approaching the macroscopic failure the strain rate presents a power law divergence with an exponent solely depending on the stress exponent $m$ of the Maxwell elements. On the microlevel the rupture process is characterized by the distribution $f(\Delta t)$ of waiting times $\Delta t$ between consecutive fiber breakings. We find that $f(\Delta t)$ has a power law behavior at any load level; however, the exponent is different on the two sides of the critical load $\sigma_{c}$, and it is a decreasing function of the stress exponent $m$.

Materials where microscopic fracture events are followed by a slow stress relaxation are, for instance, metal matrix composites where typically brittle fibers are embedded in a ductile metallic alloy [7], or biological matter where the relaxation process can also involve colloidal shear flow $[5,26]$. In short fiber random composites like paper, fiber breakings can be followed by restructuring of the surrounding fiber mat, where time dependence occurs due to frictional sliding $[2,27]$.

\section{SLOWLY RELAXING BROKEN FIBERS}

Recently, we have introduced a fiber bundle model to study the time dependent deformation and creep rupture of 


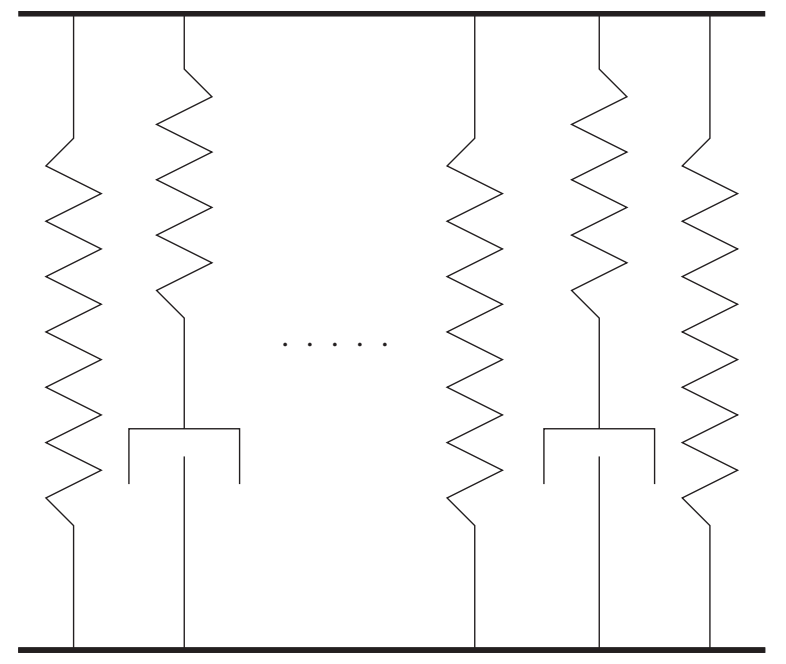

FIG. 1. The disordered solid is modeled as a parallel bundle of fibers loaded between two stiff plates. Intact fibers are assumed to be linearly elastic (springs), while the broken ones undergo a slow relaxation described by a Maxwell element (serial coupling of a spring and a dashpot).

materials. In the model, the intact fibers are linearly elastic but fiber failure is followed by a slow stress relaxation process [20]. For clarity, in this section we summarize the main steps of the model construction emphasizing aspects relevant for the present work.

In the model we consider a bundle of $N$ parallel fibers which have linearly elastic behavior with the same Young modulus $E_{f}$, so that their deformation rate can be written as

$$
\dot{\varepsilon}=\frac{\dot{\sigma}}{E_{f}} .
$$

Under a constant external load, the fibers break when the local load on them exceed their local breaking threshold $\sigma_{t h}^{i}$ which is a random variable with the probability density $p$ and distribution function $P\left(\sigma_{t h}\right)=\int_{0}^{\sigma_{t h}} p(x) d x$. When a fiber breaks we assume that its load does not drop down to zero instantaneously but the broken fiber undergoes a slow relaxation process which results in a time dependent behavior of the entire system. The broken fiber is modeled by a so-called Maxwell element which is a serial coupling of a spring and a dashpot (see Fig. 1) and it has a nonlinear response given by

$$
\dot{\varepsilon_{b}}=\frac{\dot{\sigma_{b}}}{E_{b}}+B \sigma_{b}^{m} .
$$

Here $\varepsilon_{b}$ and $\sigma_{b}$ are the time dependent strain and stress of the broken fiber, respectively. The relaxation process is characterized by the three parameters $E_{b}, B$, and $m$, where $E_{b}$ is the effective stiffness of the broken fiber and $m$ characterizes the strength of nonlinearity. The stress exponent $m$ is a very important material parameter whose value typically falls in the range $1 \leq m \leq 30$. Later on we will show that $m$ has a substantial effect on the failure process of the creeping fiber bundle both on the micro- and macrolevels. When a fiber fails, its load has to be overtaken by the remaining intact fibers. Assuming global load sharing, under an external load $\sigma_{0}$ the macroscopic constitutive equation of the system can be cast in the form

$$
\sigma_{0}=\sigma(t)\{1-P[\sigma(t)]\}+\sigma_{b}(t) P[\sigma(t)],
$$

where $\sigma(t)$ denotes the time dependent load of intact fibers, furthermore, $\{1-P[\sigma(t)]\}$ and $P[\sigma(t)]$ are the time dependent fraction of intact and broken fibers, respectively. Equation (3) expresses that the external load $\sigma_{0}$ is not only carried by the intact fibers but the broken ones also have a finite loadbearing capacity. It follows from Eq. (3) that due to the time dependent relaxation of the broken elements the load on the intact fibers $\sigma$ becomes also time dependent. We assume that the bundle is loaded between two stiff plates which ensures the boundary condition for the strain rates $\dot{\varepsilon}=\dot{\varepsilon}_{b}$. For the illustration of the model construction see Fig. 1. The equation of motion which describes the time evolution of the system can be obtained by expressing $\sigma_{b}(t)$ with $\sigma$ from Eq. (3) and substituting it into Eq. (2). Using the boundary condition of strain rates we get

$$
\begin{gathered}
\dot{\sigma}\left\{\frac{1}{E_{f}}-\frac{1}{E_{b}}\left[1-\frac{1}{P(\sigma)}+\frac{p(\sigma)}{P(\sigma)^{2}}\left(\sigma-\sigma_{0}\right)\right]\right\} \\
=B\left[\frac{\sigma_{0}-\sigma(1-P(\sigma))}{P(\sigma)}\right]^{m} .
\end{gathered}
$$

This is a first order differential equation which should be solved at a constant external load $\sigma_{0}$ for the load of intact fibers $\sigma(t)$. Since the intact fibers are linearly elastic, the macroscopic deformation-time history of the bundle can simply be obtained as $\varepsilon(t)=\sigma(t) / E_{f}$.

We have shown that for the solution $\sigma(t)$ of the equation of motion Eq. (4) and hence for the macroscopic deformation $\varepsilon(t)$ of the bundle, two regimes can be distinguished depending on the value of the external load $\sigma_{0}$ [20]: there exists a critical load $\sigma_{c}$ below which Eq. (4) has a stationary solution $\sigma_{s}$ In this load regime the bundle suffers only partial failure and relaxes to a constant deformation $\varepsilon_{s}$ (see Fig. 2). Exceeding $\sigma_{c}$, however, no stationary solution exists which implies complete breakdown at a finite time defining the lifetime $t_{f}$ of the system. Approaching the critical load $\sigma_{c}$ from above, the lifetime $t_{f}$ of the bundle has a power law divergence with an exponent which depends on $m$ but is independent of the threshold disorder of fibers [20]

$$
t_{f} \sim\left(\sigma_{0}-\sigma_{c}\right)^{-(m-1 / 2)} .
$$

In order to capture the role of the disordered microscopic strength of fibers in the rupture process, we developed an event driven Monte Carlo simulation technique which makes possible the study of finite systems up to $N=10^{8}$ fibers with moderate computational costs [20]. The simulation technique is based on the fact that due to the global load sharing the fibers break in the increasing order of their breaking thresholds $\sigma_{t h}^{i}$, where $i=1, \ldots, N$. For more details of the model and of the simulation techniques see Ref. [20]. Recently, we have also generalized the model to the case of localized load sharing of fibers after failure events [22]. In the present calculations, for the disorder distribution of the failure thresh- 


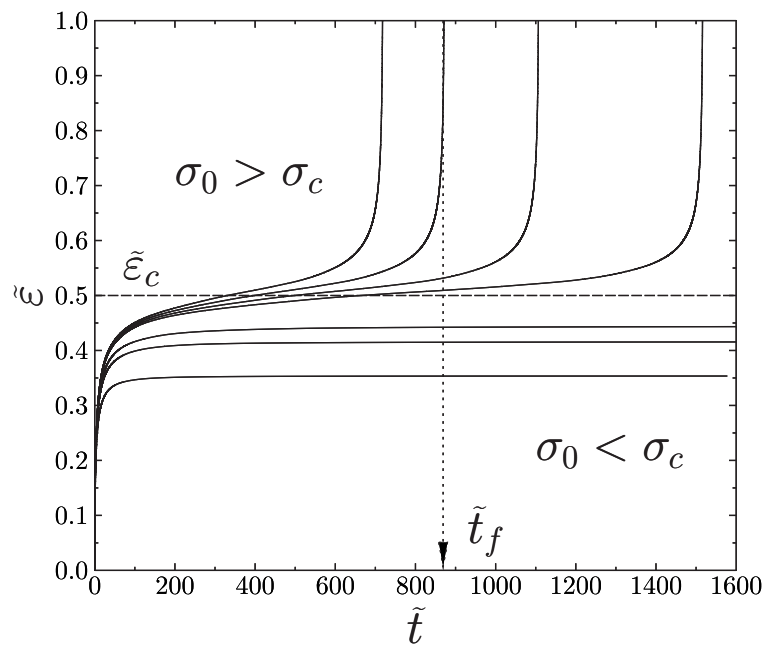

FIG. 2. $\widetilde{\varepsilon}$ as a function of $\tilde{t}$ for a bundle of $10^{7}$ fibers with uniformly distributed breaking thresholds between 0 and 1 at several values of $\widetilde{\sigma}_{o}$ below and above $\widetilde{\sigma}_{c}$. The stress exponent $m$ was set to $m=2.0$.

olds we consider a uniform distribution between 0 and 1 , and a Weibull distribution with the distribution function

$$
P\left(\sigma_{t h}\right)=1-\exp \left[-\left(\frac{\sigma_{t h}}{\lambda}\right)^{\rho}\right] \text {. }
$$

In order to vary the amount of disorder, simulations were carried out at several different values of the exponent $\rho$, while $\lambda=1$ was fixed throughout the calculations. State variables of the model system can be made dimensionless using their characteristic scale parameters. The dimensionless stress, strain, and time can be defined as $\widetilde{\sigma}=\sigma / \lambda, \widetilde{\varepsilon}=\varepsilon E_{f} / \lambda$, and $\tilde{t}=t E_{f} B \lambda^{m-1}$, respectively. In the following, the numerical results obtained by computer simulations will be presented in dimensionless form. The Young modulus characterizing the relaxing broken fibers was set to $E_{b}=E_{f} / 2$.

In the present paper, extending our former work, we focus on the process of creep rupture of the bundle of slowly relaxing fibers and characterize how the system approaches macroscopic failure on the macro and micro levels. Our analytic calculations and computer simulations of finite bundles revealed several interesting aspects of the rupture process which can be exploited in forecasting techniques of imminent failure.

\section{MACROSCOPIC BEHAVIOR}

Under a constant external load $\sigma_{0}$, the macroscopic evolution of the creeping system is characterized by the deformation $\varepsilon(t)$ and deformation rate $\dot{\varepsilon}(t)$ diagrams, which are accessible experimentally up to macroscopic failure. One of the most challenging problems for theoretical studies on creep rupture of disordered materials is to derive observables and scaling laws which allow for an accurate prediction of the lifetime of samples from short term measurements. Our computer simulations showed that the rupture life $t_{f}$ of finite bundles has large sample-to-sample fluctuations which can

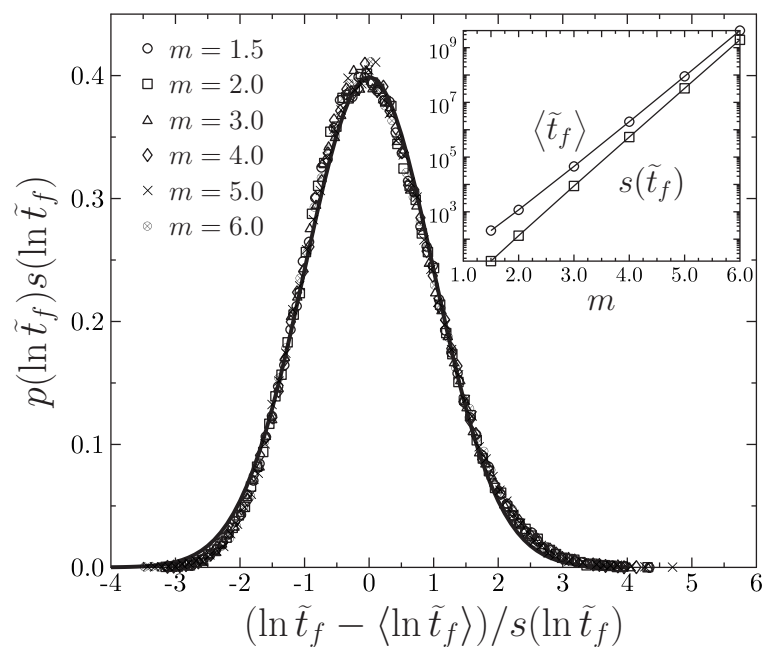

FIG. 3. Distribution of the logarithm of lifetimes $p\left(\ln \tilde{t}_{f}\right)$ rescaled by the mean $\left\langle\ln \tilde{t}_{f}\right\rangle$ and standard deviation $s\left(\ln \tilde{t}_{f}\right)$ for different values of $m$. Simulations were carried out using Weibull distributed failure thresholds with $\rho=2$. The continuous line indicates the standard Gaussian. The inset shows that with increasing $m$ both $\left\langle\ln \tilde{t}_{f}\right\rangle$ and $s\left(\ln \tilde{t}_{f}\right)$ increase exponentially. Averages were made over $10^{3}$ samples.

be attributed to the quenched disorder of fiber strength. In order to characterize these fluctuations, we determined the probability distribution of lifetime $p\left(t_{f}\right)$ which proved to have a $\log$-normal form, i.e., the logarithm of $t_{f}$ has a normal distribution with the form

$$
p\left(\ln t_{f}\right)=\frac{1}{s\left(\ln t_{f}\right) \sqrt{2 \pi}} \exp \left[-\frac{\left(\ln t_{f}-\left\langle\ln t_{f}\right\rangle\right)^{2}}{2 s^{2}\left(\ln t_{f}\right)}\right],
$$

where $\left\langle\ln t_{f}\right\rangle$ and $s\left(\ln t_{f}\right)$ denote the mean and standard deviation of the logarithmic lifetime $\ln t_{f}$. In order to numerically demonstrate the validity of Eq. (7), in Fig. 3 we plotted the standardized distribution, namely, $s\left(\ln t_{f}\right) p\left(\ln t_{f}\right)$ is presented as a function of $\left(\ln t_{f}-\left\langle\ln t_{f}\right\rangle\right) / s\left(\ln t_{f}\right)$ together with the standard Gaussian $p(x)=1 / \sqrt{2 \pi} \exp \left[-x^{2} / 2\right]$. It can be seen that an excellent agreement is obtained between the numerical results and the standard Gaussian for all the stress exponents $m$ considered. The inset of Fig. 3 demonstrates that both the mean $\left\langle t_{f}\right\rangle$ and standard deviation $s\left(t_{f}\right)$ of the rupture life increase exponentially with the stress exponent of the material. The result implies that in the case of high $m$ values relevant for experiments, large fluctuations of $t_{f}$ arise. Consequently, the lifetime estimation for finite samples requires the development of methods which provide reliable results for single samples without averaging.

Based on the evolution of the rate of deformation $\dot{\varepsilon}(t)$, the creep rupture process can be divided into three regimes: In the primary creep regime, $\dot{\varepsilon}(t)$ rapidly decreases with time which can be well approximated by a power law for a broad class of materials $\dot{\varepsilon}(t) \sim t^{-p}$ (also called Andrade law $[13,28,29])$. The secondary creep is characterized by a slowly varying, almost steady deformation rate, which is then followed by a strain acceleration in the tertiary regime 


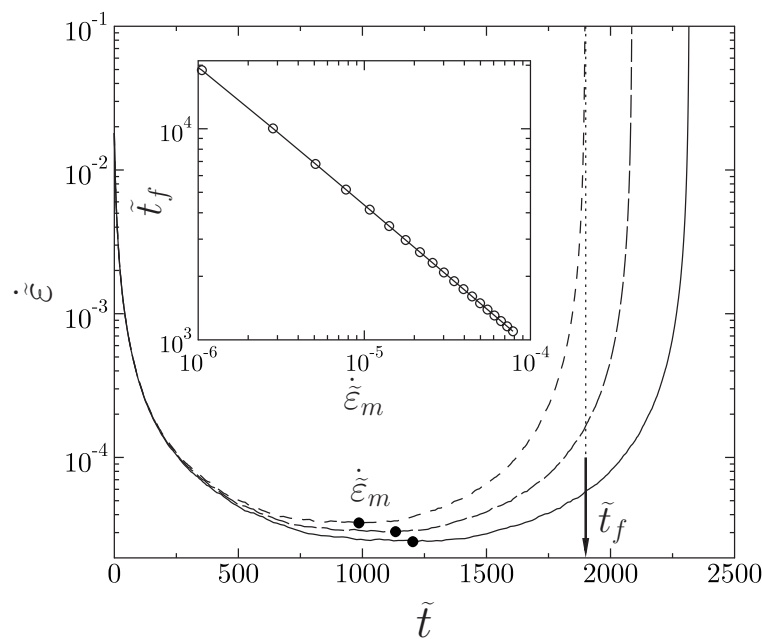

FIG. 4. Semi-log plot of the rate of deformation as a function of time for different load values in the range $\widetilde{\sigma}_{0}>\widetilde{\sigma}_{c}$ at $m=2.0$ using Weibull distributed failure thresholds with $\rho=2.0$. The inset shows a $\log$-log plot of the lifetime $\tilde{t}_{f}$ of the system as a function of the minimum strain rate $\dot{\widetilde{\varepsilon}}_{m}$. Excellent agreement is obtained with the analytic prediction Eq. (10).

for high enough external loads [6,7]. Therefore the stain rate will exhibit a minimum at a value $\dot{\varepsilon}_{m}$ as it is illustrated in Fig. 4 for different load values above the critical point. In laboratory experiments, the failure time $t_{f}$ of the specimen is usually estimated from the variation of the deformation rate $\dot{\varepsilon}$ based on the so-called Monkman-Grant (MG) relationship [30]. The Monkman-Grant relation is a semi-empirical formula which states that the time-to-failure of the system $t_{f}$ is uniquely related to the minimum creep rate $\dot{\varepsilon}_{m}$ in the form of a power law

$$
t_{f} \sim \dot{\varepsilon}_{m}^{-\xi}
$$

where the exponent $\xi>0$ depends on material properties; for metallic materials its value is close to unity $[3,6]$. The advantage of Eq. (8) is that once the relation is established from short term tests, the rupture life $t_{f}$ can be determined just following the system until the minimum of $\dot{\varepsilon}$ is reached. Since $t_{f}$ diverges when approaching the critical load from above, such estimates are of high relevance for the load regime $\sigma_{c} \lesssim \sigma_{0}$. The Monkman-Grant relation of our model system can be derived starting from the equation of motion Eq. (4): making use of the property that the term $\sigma[1$ $-P(\sigma)]$ on the right-hand side has a maximum of value $\sigma_{c}$ [31-33], the minimum deformation rate $\dot{\varepsilon}_{m}$ as a function of load can be obtained as

$$
\dot{\varepsilon}_{\mathrm{m}} \sim\left(\sigma_{0}-\sigma_{c}\right)^{m} .
$$

Numerical tests of the above relation can be seen in Fig. 5, where Monte Carlo simulations were performed with $N$ $=10^{7}$ fibers of uniformly distributed breaking thresholds varying the stress exponent $m$ of the Maxwell elements. The slope of the fitted straight lines is always equal to the corresponding value of $m$ in excellent agreement with Eq. (9).

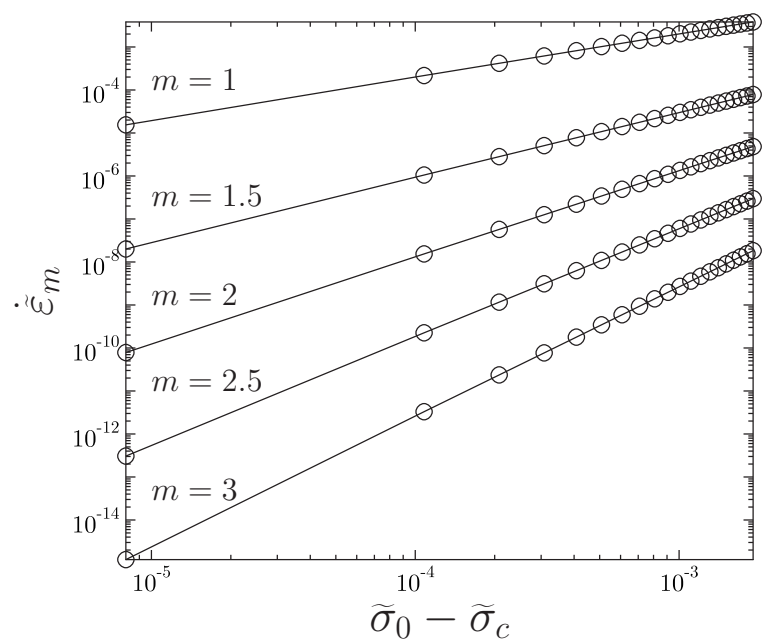

FIG. 5. Log-log plot of the minimum strain rate $\dot{\widetilde{\varepsilon}}_{m}$ as a function of $\widetilde{\sigma}_{0}-\widetilde{\sigma}_{c}$ for several values of the stress exponent $m$ with uniformly distributed breaking threshold. The slope of the fitted straight lines is equal to the specific value of $m$ in each case in agreement with Eq. (9).

Combining Eqs. (5) and (9), we obtain the lifetime as a function of the minimum strain rate

$$
t_{f} \sim \dot{\varepsilon}_{m}^{-(1-1 / 2 m)}
$$

which implies that the Monkman-Grant exponent of our model system $\xi=1-1 / 2 m$ increases from $1 / 2$ to 1 as $m$ increases starting from 1 . Representative examples of $\dot{\varepsilon}(t)$ obtained by Monte Carlo simulations at different load values $\sigma_{0}$ can be seen in Fig. 4 for $m=2.0$. The inset illustrates that the lifetime $t_{f}$ has a power law dependence on $\dot{\varepsilon}_{m}$ whose exponent coincides with the analytic prediction Eq. (10).

Besides the Monkman-Grant relation to predict lifetime of loaded specimens $t_{f}$, it has recently been pointed out that $t_{f}$ can directly be related to the characteristic time $t_{m}$ of the intermediate creep regime [18]. Here $t_{m}$ is the time where the system reaches the minimum value of the strain rate $\dot{\varepsilon}_{m}$. Experiments on different types of composites revealed that $t_{f} \approx 3 / 2 t_{m}$ holds from which $t_{f}$ can be obtained from a measurement of $t_{m}$ with a significantly shorter duration [8]. For our model, Fig. 6 presents $t_{f}$ as a function of $t_{m}$ for a uniform and a Weibull distribution of thresholds varying the stress exponent $m$. Note that each symbol in Fig. 6 represents a single sample with different realizations of the disorder and different values of the external load above the corresponding critical point $\sigma_{c}$. It can be observed that all the points fall on the same straight line with relatively small deviations implying a linear relationship

$$
t_{f}=a t_{m}
$$

with no additive term. The parameter $a$ has a universal value $a=2.05$ independent of the type of disorder and of the stress exponent $m$. At high load values (in the regime of short lifetimes in Fig. 6) the large fluctuations of the deformation rate make difficult the precise determination of $t_{m}$, which may give rise to a weak systematic deviation from the simple 


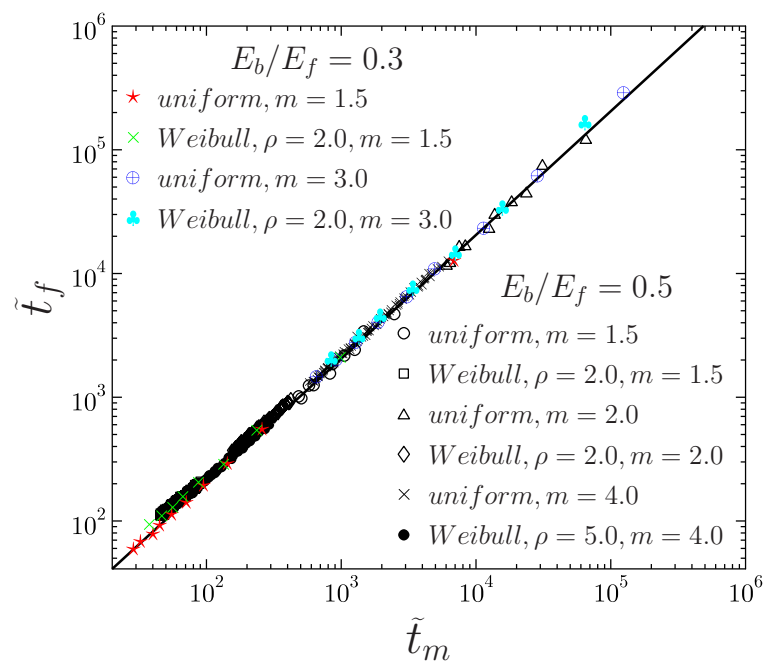

FIG. 6. (Color online) Log-log plot of the lifetime $\widetilde{t}_{f}$ of the fiber bundle as a function of the characteristic time $\widetilde{t}_{m}$ of the intermediate creep regime for different threshold distributions. A linear fit is also given. Logarithmic scales are used due to the broad ranges of $\widetilde{t}_{m}$ and $\tilde{t}_{f}$. Two sets of calculations are presented at two distinct values of the ratio $E_{b} / E_{f}$ of the Young moduli of broken and intact fibers.

linear form Eq. (11). It is important to emphasize that the results obtained at different values of the ratio $E_{b} / E_{f}$ fall on the top of each other in Fig. 6, which implies that the Young modulus of the relaxing broken fibers does not affect the qualitative behavior of the system. Note that the creep rupture model of Ref. [18] also provided $a=2.0$ with the Eyring rheology of representative volume elements. This result means that above $\sigma_{c}$ the deformation-time diagrams $\varepsilon(t)$ of the system presented in Fig. 2 are symmetric with respect to the inflection point of the curves. It is interesting to note that the fractions of fibers which break before and after the characteristic time $t_{m}$ of the intermediate creep regime, are only equal to each other when the quasistatic constitutive curve $\sigma_{0}\left(\varepsilon_{s}\right)$ of the system is a symmetric function. For instance, for the specific case of uniformly distributed breaking thresholds, the constitutive behavior of the fiber bundle is symmetric with respect to the maximum, however, it does not hold for the Weibull distribution $[25,33,34]$. The general relation of the lifetime $t_{f}$ and characteristic time $t_{m}$ Eq. (11) implies that the symmetry of the creep rupture process is independent on the symmetry properties of the constitutive curve.

Figure 4 also demonstrates that during damage enhanced creep processes, the macroscopic failure of the specimen is approached by an acceleration of the strain rate and breaking rate of fibers. An analogous effect has been observed in rupture experiments on disordered materials with increasing external load, where the acoustic emission rate has a power law singularity at catastrophic failure which also allowed for the possibility of predicting imminent failure events [2,11-13,17,35-37]. We carried out computer simulations analyzing the strain rate $\dot{\varepsilon}$ of our bundle of slowly relaxing fibers in the vicinity of the failure time $t_{f}$. The numerical calculations revealed a power law divergence of $\dot{\varepsilon}$ as a function of the distance from $t_{f}$ (see Fig. 7)

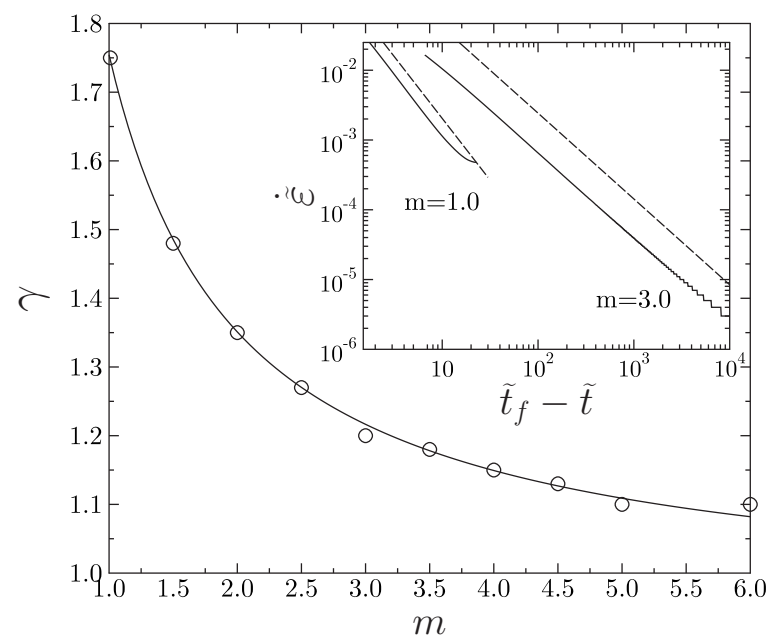

FIG. 7. The exponent $\gamma$ characterizing the divergence of the deformation rate $\dot{\widetilde{\varepsilon}}$ when approaching the time-to-failure $\tilde{t}_{f}$. Inset: Log-log plot of $\dot{\widetilde{\varepsilon}}$ as a function of $\tilde{t}_{f}-\tilde{t}$ for two different stress exponents $m$. The dashed straight lines are guides to the eye with slope 1.75 and 1.19 for $m=1$ and $m=3$, respectively. The breaking thresholds are Weibull distributed with $\rho=2.0$.

$$
\dot{\varepsilon} \sim\left(t_{f}-t\right)^{-\gamma},
$$

with an exponent $\gamma$ which does not depend on the external load $\sigma_{0}$ and on the disorder distribution, but is a decreasing function of the stress exponent $m$ governing the relaxation of broken fibers.

\section{MICROSCOPIC DYNAMICS}

On the microlevel, during the creep process the fibers break in a single avalanche which either stops and the bundle stabilizes after the breaking of a finite fraction of fibers $\left(\sigma_{0}\right.$ $<\sigma_{c}$ ), or the avalanche continues until macroscopic failure occurs $\left(\sigma_{0}>\sigma_{c}\right)$. Inside this avalanche, due to the disordered breaking thresholds, fibers may break in faster or slower sequences leading to fluctuations of the breaking rate. The waiting times $\Delta t$ between consecutive fiber breakings and their distribution characterize the microscopic evolution of the rupture process providing information also on the cascading nature of breakings. In laboratory experiments, single fiber breakings can be recorded by means of the acoustic emission techniques. Representative examples of waiting times $\Delta t$ obtained by computer simulations of the slowly relaxing fiber bundle are shown in Fig. 8 for loads below [Fig. 8(a)] and above $\sigma_{c}$ [Fig. 8(b)]. We observe that in both cases at the beginning of the creep process a large number of fibers breaks which results in short waiting times, i.e., all the $\Delta t \mathrm{~s}$ are small at the beginning. Below the critical load $\sigma$ $<\sigma_{c}$, at the macrolevel a stationary state is attained after a finite fraction of fibers broke. Approaching the stationary state $\Delta t$ becomes larger [see Fig. 8(a)] and reaches a maximum value on the plateau of $\varepsilon(t)$ (compare to Fig. 2). Above the critical load $\sigma>\sigma_{c}$, however, the slow plateau regime with long waiting times is followed by a strain acceleration (Fig. 2) accompanied by a large number of breakings result- 

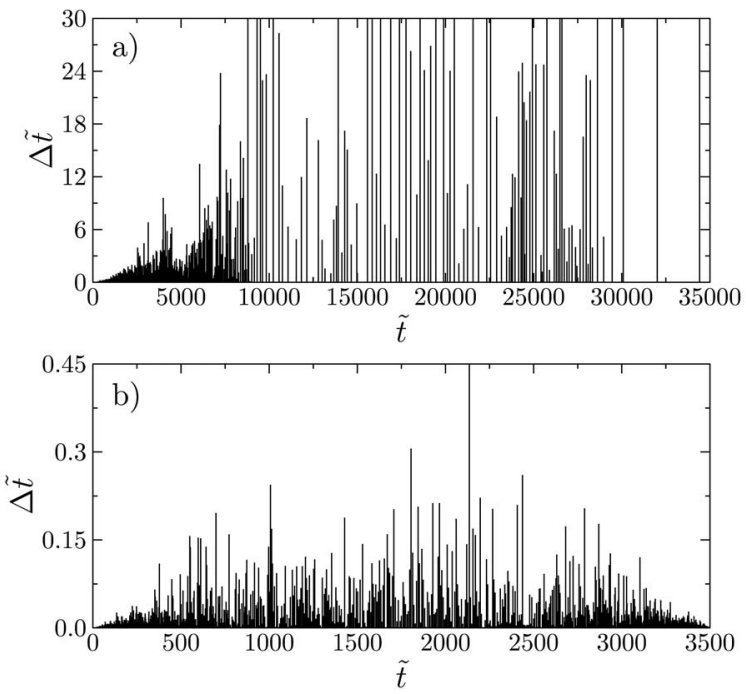

FIG. 8. Waiting times $\Delta \widetilde{t}$ between consecutive fiber breakings for two different load levels. (a) $\widetilde{\sigma}_{0}<\widetilde{\sigma}_{c}$ and (b) $\widetilde{\sigma}_{0}>\widetilde{\sigma}_{c}$ for the stress exponent $m=2.0$ with uniformly distributed breaking thresholds. For clarity, in (a) only every 10th breaking event is shown and the maximum of the vertical scale is adjusted to a relatively low value to be able to see also small $\Delta \tilde{t}$.

ing again in small $\Delta t$ s [Fig. 8(b)]. Varying the stress exponent $m$, the qualitative behavior of $\Delta t$ in Fig. 8 does not change.

Besides the overall tendencies described above, the waiting times $\Delta t$ show quite an irregular local pattern with large fluctuations and have a nontrivial distribution. We determined the distribution function $f(\Delta t)$ on both sides of the critical point $\sigma_{c}$ varying the stress exponent $m$ within a broad range. Examples of $f(\Delta t)$ are presented in Fig. 9 for $m=2.0$, where a power law form of $f(\Delta t)$ can be observed on both sides of the critical point $\sigma_{c}$. For $\sigma_{0}<\sigma_{c}$, no cutoff function

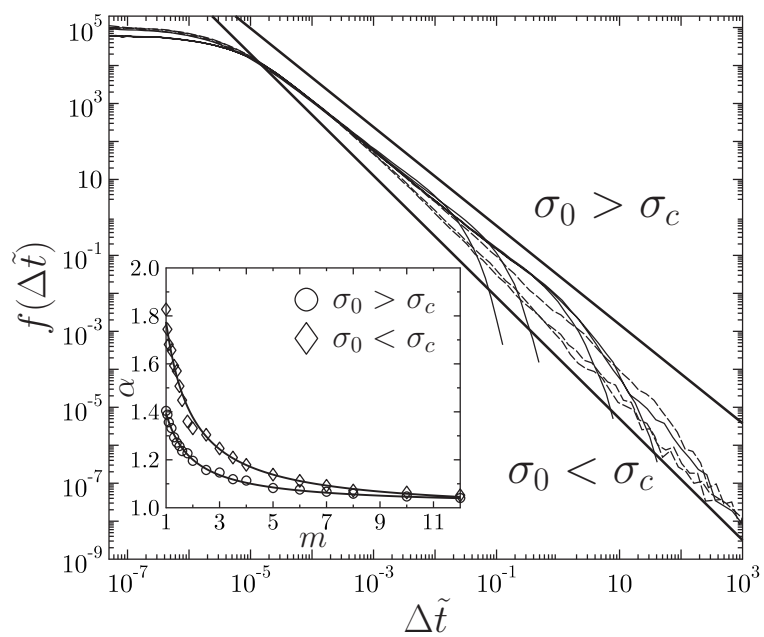

FIG. 9. Log-log plot of the distribution of waiting times $f(\Delta \widetilde{t})$ at different load levels on both sides of the critical point $\widetilde{\sigma}_{c}$ for $m$ $=2.0$ with the same disorder as in Fig. 8. Power law distributions are obtained with different exponents $\alpha$ below and above $\widetilde{\sigma}_{c}$. Inset: $\alpha$ as a function of the stress exponent $m$ below and above $\widetilde{\sigma}_{c}$.

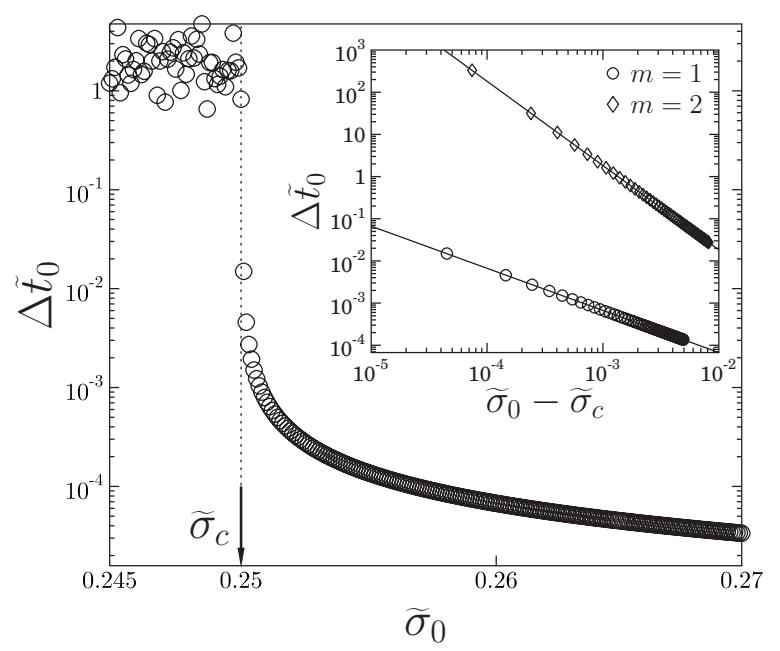

FIG. 10. The cutoff of the waiting times $\Delta \widetilde{t}_{0}$ as a function of load $\widetilde{\sigma}_{0}$ below and above the critical point for uniformly distributed thresholds with stress exponent $m=1.0$. The location of $\widetilde{\sigma}_{c}$ is indicated by the arrow. Inset: $\log -\log$ plot of $\Delta \tilde{t}_{0}$ as a function of the distance from the critical load for two stress exponents. The slope of the fitted straight lines $\beta$ is always equal to the corresponding $m$ value, in agreement with the analytic prediction.

can be identified, the power law prevails over 6-7 orders of magnitude in $\Delta t$ up to the largest values. For $\sigma_{0}>\sigma_{c}$ the power law regime is followed by an exponential cutoff which shifts to higher $\Delta t$ values when approaching the critical load from above. Hence, $f(\Delta t)$ can be cast into the functional form

$$
f(\Delta t) \sim \Delta t^{-\alpha} e^{-\Delta t / \Delta t_{0}}
$$

where the cutoff $\Delta t_{0}$ is a decreasing function of the external load $\sigma_{0}$ for $\sigma_{0}>\sigma_{c}$. The value of the exponent $\alpha$ is different on the two sides of $\sigma_{c}$ but inside one regime it is independent on the actual value of $\sigma_{0}$. It is interesting that computer simulations revealed a strong dependence of $\alpha$ on the stress exponent $m$. The inset of Fig. 9 demonstrates that with increasing $m$ the value of $\alpha$ decreases both in the under-critical and over-critical cases and tend to the same limit value $\alpha$ $\rightarrow 1$ at large $m$.

In Fig. 10 we present the cutoff $\Delta t_{0}$ as a function of $\sigma_{0}$ which was determined numerically by assuming to be proportional to the largest waiting time $\Delta t_{0} \sim \Delta t_{\max }$. It can be seen in Fig. 10 that approaching the critical load from above, $\Delta t_{0}$ rapidly increases and has a power law divergence as a function of $\sigma_{0}-\sigma_{c}$,

$$
\Delta t_{0} \sim\left(\sigma_{0}-\sigma_{c}\right)^{-\beta}
$$

which is demonstrated by the inset of Fig. 10. This behavior implies that in the limit $\sigma_{0} \rightarrow \sigma_{c}$, the exponential cutoff disappears and $f(\Delta t)$ becomes a pure power law. The cutoff exponent $\beta$ can be calculated analytically as a function of the stress exponent $m$ of the Maxwell elements: since the cutoff $\Delta t_{0}$ is proportional to the inverse of the minimum strain rate $\dot{\varepsilon}_{m}^{-1}$, it follows from Eq. (9) that 


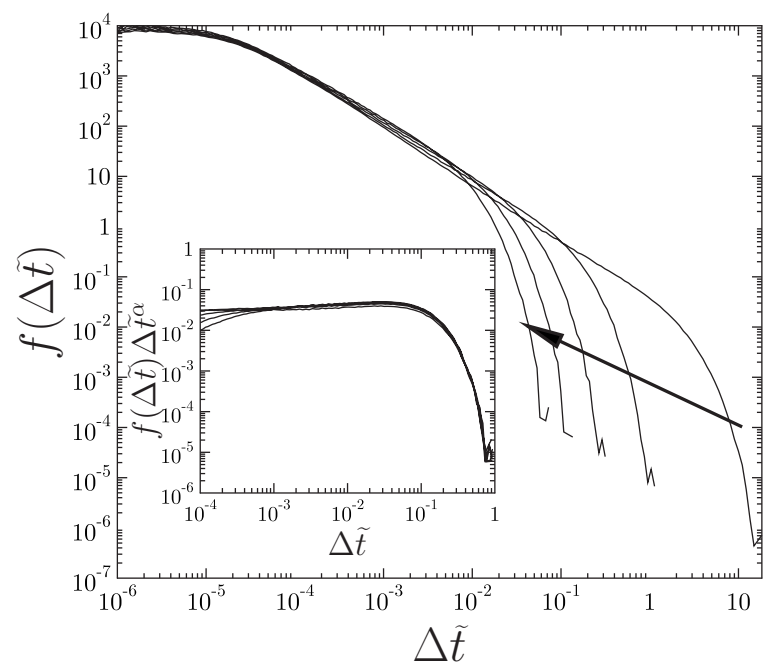

FIG. 11. Test of the validity of the functional form Eq. (13) for the distribution of waiting times $f(\Delta \widetilde{t})$ above $\widetilde{\sigma}_{c}$. The arrow indicates the increasing load. Distributions obtained at different $\widetilde{\sigma}_{0}$, are replotted in the inset by rescaling the two axis. The good data collapse confirms the validity of the scaling.

$$
\beta=m \text {. }
$$

The numerical results presented in the inset of Fig. 10 are in an excellent agreement with this analytic prediction. In order to check the validity of the functional structure Eq. (13) we carried out a data collapse of the distributions $f(\Delta t)$, i.e., plotting $f(\Delta t) \Delta t^{\alpha}$ as a function of $\Delta t / \Delta t_{0}$, the curves obtained at different values of $\sigma_{0}$ in the regime $\sigma_{0}>\sigma_{c}$ should fall on the top of each other if Eq. (13) holds. The high quality collapse obtained in Fig. 11 confirms the validity of the functional form Eq. (13).

Below the critical point $\sigma_{c}$, the distribution of waiting times does not have a cutoff function, furthermore, changing the load in this regime $\sigma_{0}<\sigma_{c}$ only reduces the statistics of the results (at lower loads less fibers break) but the functional form of $f(\Delta t)$ does not change (see Fig. 9). The behavior of $\Delta t_{0}$ as a function of $\sigma_{0}$ in Fig. 10 shows that for $\sigma_{0}<\sigma_{c}$ the system is always in the state of $\Delta t_{0} \rightarrow \infty$ so that in Eq. (13) a pure power law remains. Note that the finite value of $\Delta t_{0}$ in Fig. 10 is due to the finite system size.

\section{DISCUSSION AND OUTLOOK}

We presented a detailed study of the creep rupture process of disordered materials focusing on observables which can be relevant to forecast the failure event. Analytical calculations and computer simulations were carried out in the framework of a fiber bundle model for creep rupture where time dependence is introduced by the slow relaxation of broken fibers represented by Maxwell elements. The intact fibers have linearly elastic behavior up to failure with identical Young modulus but random breaking thresholds. The most important dynamic parameter of the model is the stress exponent $m$ of the Maxwell elements, characterizing the strength of nonlinearity in the relaxation process.
We showed that above the critical load the bundle suffers macroscopic failure at a finite time, which diverges when approaching the critical point. Due to the strength disorder of fibers, the lifetime of the bundle has strong sample-to-sample fluctuations described by log-normal distributions independent of the type of disorder. The mean and standard deviation of $t_{f}$ increase exponentially with the stress exponent, which requires forecasting techniques of the imminent failure based on measurements of single samples. We derived the Monkman-Grant relation of the model, i.e., we proved analytically that the lifetime of the system has a power law dependence on the minimum strain rate, and additionally, we showed that a universal relation exists between the characteristic time of the intermediate creep regime and the lifetime of the system. Both relations allow for reliable lifetime estimates of samples based on short term measurements. Approaching macroscopic failure, the deformation rate proved to have a power law divergence with an exponent depending on $m$.

On the microlevel, the failure process is characterized by the distribution of waiting times between consecutive fiber breakings, which has a power law behavior on both sides of the critical load but with different exponents. Computer simulations revealed that the exponents are decreasing functions of the stress exponent $m$, furthermore, the cutoff waiting time has a power law divergence when approaching the critical point from above. The cutoff exponent was found to be equal to the stress exponent of Maxwell elements $m$.

Our model calculations give a reasonable description of the time dependent response, for instance, of strongly bonded long fiber metal matrix composites, where fiber failure is followed by a slow relaxation due to the yielding matrix [7]. A qualitatively similar relaxation process occurs also in bio-materials after local rupture events $[5,26]$ and in random mats of short fibers with frictional contacts (paper, textile) $[2,27,38,39]$. In metal matrix composites, the stress exponent typically has high values $m>10$. Hence, in these cases our calculations predict for the Monkman-Grant exponent $\xi \rightarrow 1$, for the exponent of the diverging strain rate $\gamma$ $\rightarrow 1$, for the exponent of the waiting time distribution $\alpha$ $\rightarrow 1$ on both sides of $\sigma_{c}$, while the cutoff exponent $\beta$ takes large values $\beta=m$. Recent experiments on various types of fracture processes revealed a power law distribution of waiting times between consecutive local breaking events $[15,18,40-42]$. The measured value of the exponent $\alpha$ typically falls between 1.0 and 2.0 in good agreement with our model calculations. We propose further experimental tests of our theoretical predictions.

\section{ACKNOWLEDGMENTS}

This work was supported by the Spanish-Hungarian Intergovernmental Project No. HH-2005-0015. K.K., S.N., and F.K. were also supported by OTKA T049209 and NKFP-3A/ 043/04. R.C.H. acknowledges the financial support of the Spanish Minister of Education and Science, through a Ramon y Cajal Program. I.P. and R.C.H. acknowledge financial support from DGICYT of the Spanish Government (FIS2005-01299). I.P. thanks Distinció from DURSI (Generalitat de Catalunya). 
KOVÁCS et al.

[1] Statistical Models for the Fracture of Disordered Media, edited by H. J. Herrmann and S. Roux, Random Materials and Processes (Elsevier, Amsterdam, 1990).

[2] M. Alava, P. K. Nukala, and S. Zapperi, Adv. Phys. 55, 349 (2006).

[3] Damage and Its Evolution in Fiber-Composite Materials, edited by G. Busse, B.-H. Kröplin, and F. K. Wittel (ISD Verlag, Stuttgart, 2006).

[4] T. Erdmann and U. S. Schwarz, Phys. Rev. Lett. 92, 108102 (2004).

[5] B. G. Yoon, J. Choi, and M. Y. Choi, J. Korean Phys. Soc. 47, 1053 (2005).

[6] K. K. Chawla, Composite Materials. Science and Engineering, Materials Research and Engineering (Springer, New York/ Berlin, 1987).

[7] R. M. Jones, Mechanics of Composite Materials, 2nd ed. (Taylor \& Francis, Philadelphia/London, 1999).

[8] H. Nechad, A. Helmstetter, R. E. Guerjouma, and D. Sornette, J. Mech. Phys. Solids 53, 1099 (2005).

[9] B. K. Chakrabarti and L. G. Benguigui, Statistical Physics of Fracture and Breakdown in Disordered Systems (Oxford University Press, New York, 1997).

[10] K. Z. Nanjo and D. L. Turcotte, Geophys. J. Int. 162, 859 (2005).

[11] A. Johansen and D. Sornette, Eur. Phys. J. B 18, 163 (2000).

[12] J.-C. Anifrani, C. L. Floch, D. Sornette, and B. Souillard, J. Phys. I 5, 631 (1995).

[13] A. Saichev and D. Sornette, Phys. Rev. E 71, 016608 (2005).

[14] A. Garcimartín, A. Guarino, L. Bellon, and S. Ciliberto, Phys. Rev. Lett. 79, 3202 (1997).

[15] A. Petri, G. Paparo, A. Vespignani, A. Alippi, and M. Costantini, Phys. Rev. Lett. 73, 3423 (1994).

[16] A. Guarino, A. Garcimartin, and S. Ciliberto, Eur. Phys. J. B 6, 13 (1998).

[17] S. Pradhan, A. Hansen, and P. C. Hemmer, Phys. Rev. Lett. 95, 125501 (2005).

[18] H. Nechad, A. Helmstetter, R. El Guerjouma, and D. Sornette, Phys. Rev. Lett. 94, 045501 (2005).

[19] R. C. Hidalgo, F. Kun, and H. J. Herrmann, Phys. Rev. E 65, 032502 (2002).

[20] F. Kun, R. C. Hidalgo, H. J. Herrmann, and K. F. Pal, Phys.
PHYSICAL REVIEW E 77, 036102 (2008)

Rev. E 67, 061802 (2003).

[21] F. Kun, Y. Moreno, R. C. Hidalgo, and H. J. Herrmann, Europhys. Lett. 63, 347 (2003).

[22] R. C. Hidalgo, F. Kun, and H. J. Herrmann, Physica A 347, 402 (2005).

[23] T. Baxevanis and T. Katsaounis, Phys. Rev. E 75, 046104 (2007)

[24] S. Pradhan and P. C. Hemmer, Phys. Rev. E 75, 056112 (2007).

[25] S. Pradhan and B. K. Chakrabarti, Int. J. Mod. Phys. B 17, 5565 (2003).

[26] B. J. Kim, Europhys. Lett. 66, 819 (2004).

[27] M. J. Alava and K. J. Niskanen, Rep. Prog. Phys. 69, 669 (2006).

[28] E. N. da C. Andrade, Proc. R. Soc. London, Ser. A 84, 1 (1910).

[29] A. Saichev and D. Sornette, Phys. Rev. E 71, 016608 (2005).

[30] F. C. Monkman and N. J. Grant, Proc. ASTM 56, 593 (1956).

[31] M. Kloster, A. Hansen, and P. C. Hemmer, Phys. Rev. E 56, 2615 (1997).

[32] J. V. Andersen, D. Sornette, and K. Leung, Phys. Rev. Lett. 78, 2140 (1997).

[33] F. Kun, S. Zapperi, and H. J. Herrmann, Eur. Phys. J. B 17, 269 (2000).

[34] Y. Moreno, J. B. Gomez, and A. F. Pacheco, Phys. Rev. Lett. 85, 2865 (2000)

[35] S. Pradhan and B. K. Chakrabarti, Phys. Rev. E 65, 016113 (2001).

[36] S. Pradhan and A. Hansen, Phys. Rev. E 72, 026111 (2005).

[37] F. Raischel, F. Kun, and H. J. Herrmann, Phys. Rev. E 74, 035104(R) (2006).

[38] R. Chudoba, M. Vorechovsky, and M. Konrad, Int. J. Solids Struct. 43, 413 (2006).

[39] M. Vorechovsky and R. Chudoba, Int. J. Solids Struct. 43, 435 (2006).

[40] L. I. Salminen, A. I. Tolvanen, and M. J. Alava, Phys. Rev. Lett. 89, 185503 (2002).

[41] S. Deschanel, L. Vanel, G. Vigier, N. Godin, and S. Ciliberto, Int. J. Fract. 140, 87 (2006).

[42] C. Maes, A. Van Moffaert, H. Frederix, and H. Strauven, Phys. Rev. B 57, 4987 (1998). 